\title{
Improving Printed Angle Sensor Performance by Compression- Tension Sensor Pair Compensation
}

This paper was downloaded from TechRxiv (https://www.techrxiv.org).

\section{LICENSE}

CC BY-NC-SA 4.0

SUBMISSION DATE / POSTED DATE

20-10-2021 / 21-10-2021

\section{CITATION}

Türkmen, Dila; Acer Kalafat, Merve (2021): Improving Printed Angle Sensor Performance by CompressionTension Sensor Pair Compensation. TechRxiv. Preprint. https://doi.org/10.36227/techrxiv.16836733.v1

$\mathrm{DOI}$ 


\section{Improving Printed Angle Sensor Performance by Compression-Tension Sensor Pair Compensation}

This work has been submitted to the IEEE for possible publication. Copyright may be transferred without notice, after which this version may no longer be accessible.

\author{
$1^{\text {st }}$ Dila Türkmen* \\ Mechanical Engineering Department \\ Istanbul Technical University \\ Istanbul Turkey \\ turkmend@itu.edu.tr
}

\author{
$2^{\text {nd }}$ Merve Acer Kalafat \\ Mechanical Engineering Department \\ Istanbul Technical University \\ Istanbul Turkey \\ acerm@itu.edu.tr
}

\begin{abstract}
Silver nanoparticle angle sensors are inkjet printed on a flexible PET substrate and implemented as pairs of compression and tension side folding individuals into a rigid experimental setup. Resulting combined signals improved the individual sensor responses due to their compensating characteristics, and gave the best performances in the existing literature in terms of linearity, sensor life-time, static and cyclic drift, hysteresis and dynamic dependency. Proposed method is promising for eliminating the major limitations on the printed sensor use in flexible hinges and paving the way to fully soft all-integrated foldable robots.
\end{abstract}

Index Terms-Printed sensors, angle sensors, flexible sensors, foldable robotics

\section{INTRODUCTION}

$\mathbf{P}$ Rinted sensors technology is promising for integrated actuation and proprioception implementations in foldable robotics, as it serves to the main goal of the soft robotics field by providing a low-cost, fully soft and easy-to-fabricate solution [1]-[4]. Angle proprioception is a major function desired in foldable robots for cases such as reconstruction (e.g. exploration) [5]-[7], set-point control (e.g. reconfiguration, pick \& place, gait control) [4], [6], [8]-[11] or tracking control (e.g. micromanipulation) [11]-[13]. Resistive sensors are widely used in deformation sensing practices such as straingauges or bend sensors ( [2], [5], [7], [14]-[16]). However their usage in foldable robots as angle sensors is limited due to their fragile performances arising from the deformation (i.e. cracks) induced working mechanism [3], [9], [11], [17], [18]. This results with many foldable robots to work in open loop or employ hard component sensor assembly (e.g. photoresistor [9], slider \& potentiometer [13]) [3]. Major drawbacks of the printed angle sensors are, nonlinearity [5], [10], [11], hysteresis [7], [10], [11], cyclic and static drifts [6], [10], [11], noise [3], [6], low repeatability and reproducability [3], [9], short life-time [11], [17] and dynamic dependency [10], [11].

Sun et al. reports the only printed angle sensor with a linear response in the literature [6]. However results were reported only between $0-60^{\circ}$ of the joint and only in the

This work was supported by the Scientific and Technological Research Council of Turkey (TUBITAK) (Grant No. 216M193).

* Corresponding author. folding direction. Authors also noted that a more complex relationship could be revealed by further inspection. Due to the irregularities in low angle response and low signal to noise ratio (SNR), estimation errors up to $10-15^{\circ}$ were recorded. Steps responses showed $2^{\circ}$ static drift. Three layers carbon inkjet printed sensors were integrated into paper foldable mechanisms with a fixed-sliding(guided) configuration.

Kwak et al. used screen printed conductive polymer and contact resistance changes to measure the hinge positions [5]. Although this sensors did not yield a regular cyclic drift as many resistive sensors do, irreguralar drift between the cycles caused estimation errors up to $3^{\circ}$. This sensors required two separate calibration lines in $0-90^{\circ}$ range, giving a total $3.3^{\circ}$ RMS accuracy.

Firouzeh et al. used carbon screen printed sensors for the gait control of an all-integrated robogami structure [10]. Linear scores were cut on the substrate prior to the printing step to increase the repeatability. $1.1^{\circ}$ accuracy was achieved at set point estimations for 50 repetitions in $0-180^{\circ}$ range. However significant static and cyclic drift behaviours were observed and estimation models were proposed. Sensors also showed nonlinearity and hysteresis.

In our previous study we reported all-silver-inkjet-printed angle sensors, embedded in a paper foldable Delta mechanism [11]. The most linear design in the study had up to $8^{\circ}$ estimation errors for a linear calibration function and required a cubic calibration function for $1.1^{\circ}$ error. A significant regular cyclic drift was reported $(1 \%$ cycle) with a static drift below $2^{\circ}$. Sensors were found to loose their sensitivity at about 300 cycles.

All of the presented literature used the printed sensors as single per joint and in compression side folding direction. Beisteiner et al. reported the opposite signed linear responses of inkjet printed patterns to tension and compression strains. However given deformations were translational [19]. Zlebic et al. attached two printed strain-gauges on the tension and compression faces of a bending cantilever beam, and measured a linear resistance change w/to strain with a Wheatstone bridge circuit [16]. However the deformations were in microstrain level and individual responses of the sensors were not reported. 
Asri et al. reported a linear response for the tension side $90^{\circ}$ folding and a parabolic response for compression side $90^{\circ}$ folding of the silver inkjet printed sensors printed on a PET substrate. However flexible sensors were not embedded into a joint (resulting with an uncertain center of rotation through the motion) and rotations into two directions were applied successively on the same sample, instead of testing separately [20].

In our preliminary observations on tension and compression side folding printed sensors (fabricated and tested together or separately), we detected that the two configurations of the sensors were giving vertically symmetrical angle responses, pointing out a possible compensating behavior [11], [21].

In this study we propose a hybridization approach where a compression and tension side folding silver inkjet printed sensor pair is embedded into a 3D printed rigid experimental setup, and used to compensate each other for their imperfections such as nonlinearity and drift. An average function is used to combine the individual sensor signals. A nonlinear function and regression tree model is also demonstrated to introduce the further capabilities of the combined approach. Sensor printed PET layers are tested in $0-90^{\circ}$ range. Proposed approach significantly eliminated the major problems seen in the individual printed sensor responses and reduce the noise and disturbance effects without any pre- or post-processing. Resulting sensing performances extended the sensing capabilities of printed sensors, to a $0.6^{\circ}$ RMS nonlinearity error, $5000+$ cycles of sensor life-time, $0.2^{\circ}$ static drift, $4 \mathrm{e}-4^{\circ} /$ cycle cyclic drift and $0.5^{\circ}$ hysteresis error.

Used fabrication methods and experimental procedure is introduced in the methods section. Combination effects on the sensor nonlinearity, static and cyclic drift, noise and disturbance cancellation, dynamic and static hysteresis and dynamic dependency compensation are presented in the results and discussion section. Individual pair results are given together with the statistics obtained from a 22 pairs experiment population. Results showed the compensation performance is reproducible among varying design parameters. Population data is provided as supplementary material.

\section{Methods}

\section{A. Fabrication}

Samples are printed using an Epson L350 office type desktop inkjet printer and silver nanoparticle (Ag NP) ink (NovaCentrix JS-B25p) on a $140 \mu \mathrm{m}$ PET substrate (Novelle). Printing parameters are set as; colored, highest quality, Epson premium glossy photo-paper. High speed print option is checked. A low-cost $(<150 \$)$ desktop laminator, Olympia A 3048 , is used to cure the printed samples. This method was found to give a faster and more consistent curing performance, compared with the conventional oven curing in our previous study [21]. $250 \mu \mathrm{m}$ heating level and 3 lamination cycles (number of pass) are used as suggested in [21], with $120 \mu \mathrm{m}$ bottom and $210 \mu \mathrm{m}$ top protective paper layers.
TABLE I

EXPERIMENT POPULATION

\begin{tabular}{cccccc|cccccc}
$\mathrm{n}$ & $\mathrm{p}$ & $\mathrm{d}$ & $\mathrm{r}$ & $\mathrm{s}$ & $\mathrm{h}$ & $\mathrm{n}$ & $\mathrm{p}$ & $\mathrm{d}$ & $\mathrm{r}$ & $\mathrm{s}$ & $\mathrm{h}$ \\
\hline 5 & TRA & 3 & 1 & $\mathrm{f}$ & 0 & 1 & TRA & 3 & 1 & $\mathrm{~s}$ & 0 \\
1 & TRA & 2 & 1 & $\mathrm{f}$ & 0 & 1 & TRA & 3 & 3 & $\mathrm{~s}$ & 0 \\
1 & TRA & 3 & 1 & $\mathrm{f}$ & -0.06 & 2 & AXL & 0.5 & 1 & $\mathrm{f}$ & 0 \\
1 & TRA & 3 & 1 & $\mathrm{f}$ & 0.4 & 2 & AXL & 1 & 1 & $\mathrm{f}$ & 0 \\
1 & TRA & 3 & 1.5 & $\mathrm{f}$ & 0 & 2 & AXL & 2 & 1 & $\mathrm{f}$ & 0 \\
1 & TRA & 3 & 2 & $\mathrm{f}$ & 0 & 2 & AXL & 3 & 1 & $\mathrm{f}$ & 0 \\
1 & TRA & 3 & 3 & $\mathrm{f}$ & 0 & 1 & AXL & 0.5 & 1 & 3 & 0 \\
n: number of sample pairs. p: pattern. s: support type & & &
\end{tabular}

\section{B. Experiments}

To increase the sensor life-time w/to the previous implementation of $\mathrm{Ag} \mathrm{np}$ inkjet printed angle sensors [11], patterns are designed to have a sufficient distance from the flexible layer edges, as these regions are more prone to deformation (Fig. 1). Sensor line width parameter (d, Fig. 1) was increased up to $3 \mathrm{~mm}$, to deal with long cracks occurring in the tension side folding [11]. However this resulted with low sensor resistances $(\sim 2 \Omega)$ and a reference resistance of $20 \Omega$ is used for the voltage divider measurement circuit, to meet the maximum current limit $(500 \mathrm{~mA})$. 5V voltage is supplied to the circuit via Arduino Uno board using the PC power output.

In order to examine how robust is the proposed compensation approach against the sensor design parameters, a set of experiment samples are fabricated varying in terms of the sensor pattern (TRA, AXL), print width (d), top rigid distance to joint axis (r), rotating link guiding type (i.e. flexible layer support type: fixed or sliding [11]) and neutral axis to center of rotation distance (h) (Fig. 1). Total number of the experiment set population is 44 ( 22 pairs) (Table I).

Three trajectories are used to test the sensor performances: A triangular wave between $0-90^{\circ}$ with a $10 \mathrm{sec}$ period is used to examine the cyclic drift, dynamic hysteresis and nonlinearity of the sensors. Two stepwise trajectories between the same full range are used to test the transient and steady state characteristics such as static drift, static hysteresis and static nonlinearity. Two stepwise trajectories differ in terms of their acceleration profiles, in order to inspect the acceleration dependency (dynamic dependency) seen in the sensor responses.

Sensor resistance and encoder angle values are normalized between 0 and 1, w/to their maximum changes at the first cycle $(\bar{R}, \bar{\theta})$. Percentage cyclic drift is also calculated relative to the same resistance change. Percentage static drift is calculated relative to the resistance change at the corresponding step. At each step drift is measured from the end of the setling $\left(t_{s}=3 \mathrm{sec}\right)$ to the end of the step time $\left(t_{\text {step }}=5 \mathrm{sec}\right)$. All measurements are taken at $20 \mathrm{~Hz}$ sampling frequency.

\section{Compression-Tension Sensor Pair}

Although an ideal implementation of compression and tension side folding sensor pair would be double side printing, existing coating technology does not allow a double side printable substrate for office type inkjet printers due to the sticking of the films to each other during fabrication (chemical coating 


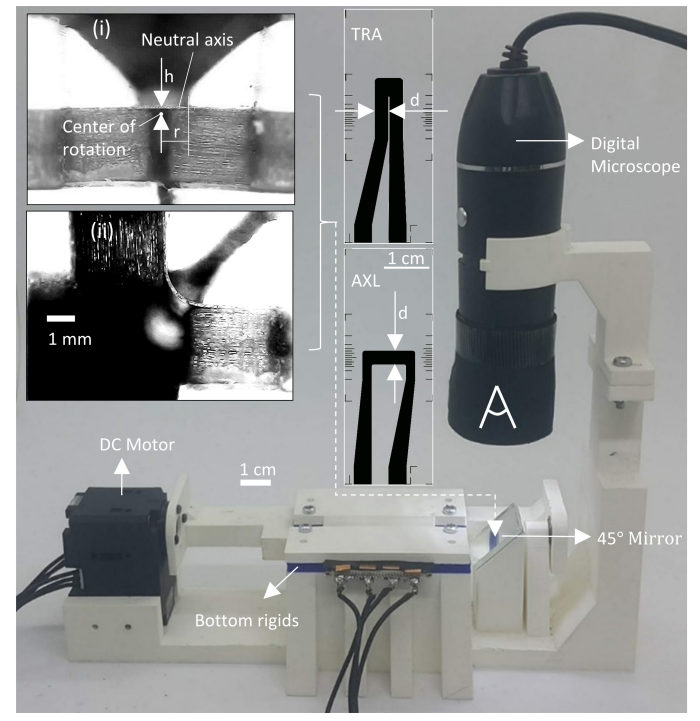

Fig. 1. Experimental setup. i and ii are sample images taken with the digital microscope using the attached mirror, at the flat and $90^{\circ}$ folded positions of the sensor printed flexible PET layer. Two patterns employed in the experiments (TRA and AXL) are also represented.

on the substrate to trigger the curing of conductive inks). For further studies, more advanced inkjet printers (e.g. Dimatix) can be employed, compatible with double side printable ink \& substrates.

After patterns are printed and cured, sensors are cut by hand using the guidelines shown in Fig. 1. Two samples of the same pattern are assembled side by side, with the conductive faces looking at opposite sides (Fig. 2). Connection between the sensors and the measurement circuit is provided with a modified FFC connector (similar to [11]) using $60 \mu \mathrm{m}$ copper sheets to evenly secure flexible layers into the FCC and enable conductivity (Fig. 2).

A 3D printed setup (Fig. 1, 2) is used to fix the two adjacent flexible layers between the top and bottom rigid plates. No adhesive layers are used as the mechanical pressure provided with the screws was sufficient to avoid any slip. Moreover this approach eliminates the uncertainty and viscous behaviour added by the adhesive layers. Along with this fixed-guided(fixed) configuration, a fixed-guided(sliding) configuration is also tested by introducing a gap between the moving link plates by plate design, which allows the PET layer freely slide between the rigid layers at the rotating link side.

\section{RESUlTS AND Discussion}

\section{A. Nonlinearity Compensation}

Matching with our preliminary observations, sensor pair angle responses showed a vertical symmetry, with respect to a curve $s(\bar{\theta})$, which is approximately equal to the encoder response (i.e. $s(\bar{\theta}) \cong \bar{R}(\bar{\theta})=\bar{\theta}$ ) (Fig. 3 (a.i)). This approximation defines the relation between the pair responses as;

$$
\bar{R}_{T}=s(\bar{\theta})+\left(s(\bar{\theta})-\bar{R}_{C}\right)=2 \cdot \bar{\theta}-\bar{R}_{C}
$$

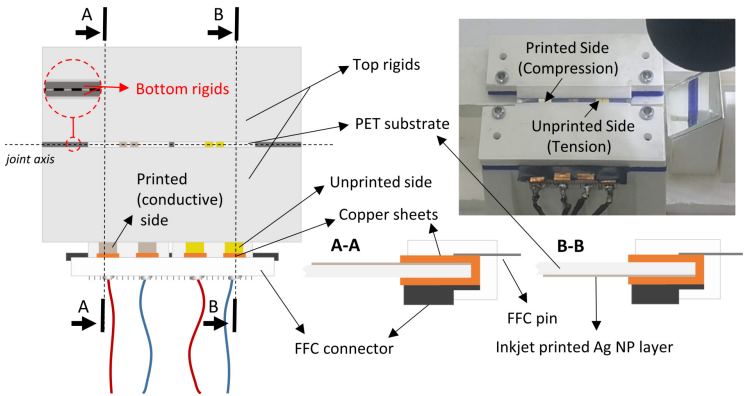

Fig. 2. Schematic and actual representation of the compression \& tension sensor pair, integrated into the experimental setup.
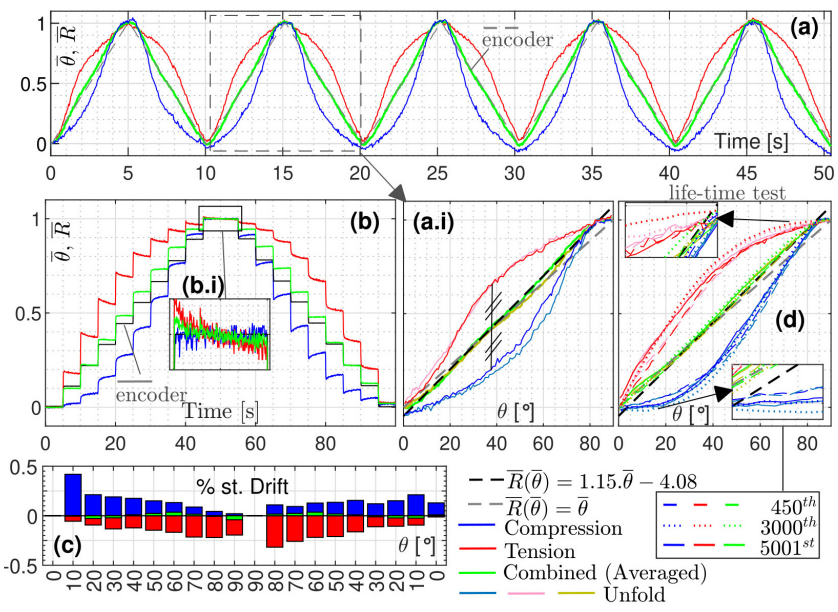

Fig. 3. Compensation performance of TRA-d3mm-r1mm-fixed-h0mm configuration pair. Data in (a) belongs to the $31-35^{t h}$ cycles of a sample pair. Data in (b-d) belongs to a separate sample pair of the same configuration, with (b) representing the stepwise trajectory response after 2400 triangular cycles, and (c) the static drifts at each step.

where $\mathrm{T}$ and $\mathrm{C}$ stands for tension and compression sensors. Thus, in order to obtain linear signal w/to angle change $\left(\bar{R}_{C o m b} \stackrel{!}{=} \bar{\theta}\right)$, a combined normalized resistance, $\bar{R}_{C o m b}$, is calculated by averaging the pair signals as;

$$
\bar{R}_{C o m b}=\frac{\bar{R}_{T}+\bar{R}_{C}}{2}
$$

Green curves in Fig. 3 shows this combined signal, which also corresponds to the experimental $s(\bar{\theta})$ curve. Although a slight mismatch exist between the encoder response and the combined signal arising from the low slope regions around $0^{\circ}$ and $90^{\circ}$, nonlinearities are compensated as expected. Fitting a line to the experimental $s(\bar{\theta})$ (Fig. 3 (a.i)), a more accurate response for the combined signal is found with,

$$
s(\bar{\theta}) \cong \bar{R}(\bar{\theta})=1.15 \cdot \bar{\theta}-4.08
$$

Although the individual response shapes are observed to differ between different configurations (pattern, $d, r, h$ ), the compensation performance is found to be highly repeatable with the same calibration lines for all. Averaging the experiment population performance for fixed configuration sample 
pairs $(\mathrm{n}=19)$, the RMSE between the combined signals and $R(\bar{\theta})=\bar{\theta}$ line is found to drop by $70 \%$ (10\% standard deviation $(\sigma)$ ) relative to the average individual response RMSE. $100 \%$ of the fixed configuration samples showed nonlinearity improvement while no symmetry and no compensation is observed in the sliding configuration samples $(n=3)$. For the latter, both the tension and compression side sensor responses showed the same behaviour, similar to the tension profile of the fixed samples.

The average RMSE obtained with the combined signals is $3.6^{\circ}\left(\sigma=0.9^{\circ}\right)$ with the $R(\bar{\theta})=\bar{\theta}$ calibration and $1.44^{\circ}$ with $R(\bar{\theta})=1.15 \cdot \bar{\theta}-4.08$. The latter calibration function gave an even lower error ( $\left.1^{\circ} \mathrm{RMSE}\right)$ when the operation range is taken as $5^{\circ}-83^{\circ}$. This constitutes the lowest nonlinearity achieved for the widest operating range within the printed angle sensor literature. Fig. 3 shows the behaviour of the samples for TRAd3mm-r1mm-fixed-h0mm configuration, which has the same RMSE with the population average.

Uniqueness and lengthened sensor life-time: Beside the calibration easiness, one other major advantage of the decreased nonlinearity is the increased sensitivity. A typical individual sensor response shows diminished sensitivity at $0^{\circ}$ and $90^{\circ}$ regions for the compression and tension types respectively (Fig. 3). However, the combined signal provides a constant and high sensitivity at almost full range (minimal insensitive areas may occur around the approximately $5^{\circ}$ neighborhoods of 0 and $90^{\circ}$ positions). This improvement brings a remarkable advance to the life-time of the sensors. In our previous study [11], same type of sensors were found to start loosing their sensitivities at around the $300^{\text {th }}$ cycle. A 9 times increase in the individual sensor life-time in the present study $(\sim 3000$ cycles, Fig. 3(d)) is due to the increased line width and pattern distance from the flexible layer edges.

Figure 3(d) shows that the combined approach took the lifetime of the sensors to much beyond, by remaining the same high sensitivity of the earlier cycles valid at the $3000^{t h}$ cycle, whereas the compression sensor lost its uniqueness between $~$ $0-15^{\circ}$ and become inoperable. Combined signals are observed to still preserve their high sensitivity at the $5000^{\text {th }}$ cycle where the experiments were cut. Fig. 3(d) shows the 5001 ${ }^{\text {st }}$ cycle which was applied the day after the 5000 cycle test. Notice the individual sensors slightly regained their sensitivity. This recovery observation is matching with the recoverable drift behaviour reported in [10]. 50\% of the fixed configuration sensor pair population showed a full range uniqueness with the combined signal at their $32^{\text {nd }}$ cycles, although none of the individual signals were unique.

\section{B. Static Drift Compensation}

Sensors showed a similar static drift to [11] where the same kind of sensors were implemented into a paper foldable robot. Drifts occured in a manner that the sensor resistances fall after a step input, independent from the folding direction at both tension and compression sensors (Fig. 3(b)). Drift amplitudes were mostly equal through the folding range and loading sides. (Fig. 3(b.i) shows a close-up to one of the exceptions.) Folding
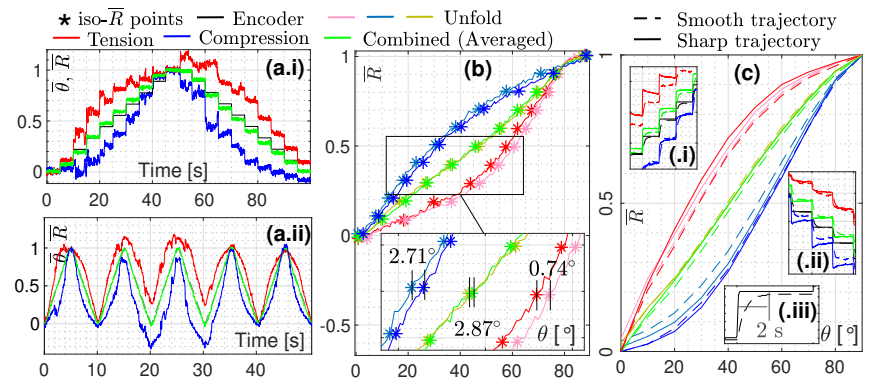

Fig. 4. (a.i) and (a.ii) represents the noise and disturbance cancellation on a AXL-d2mm-r1mm-fixed-h0mm sample pair. (b) Dynamic hysteresis compensation (sample configuration: TRA-d3mm-r2mm-fixed-h0mm). (c) Dynamic dependency compensation of a TRA-d3mm-r1mm-fixed-h0mm sample pair.

direction independency matches with the observations in [10], where the behaviour was explained with the relief of the lateral strain in the ink matrix, which formerly caused the temporary resistance increase by breaking the conductive paths.

The same signed resistance drifts in the compression and tension sensors become opposite signed when the signals are normalized between 0-1 and compensate each other when averaged for the combined signal (2). Fig. 3(c) shows the negative signed drifts through the stepwise trajectory in (b), in terms of $\%$ drifts. The compression and tension sensor signals with $14.8 \%$ and $15.8 \%$ mean drifts built up a sensor response with only $2 \%\left(0.2^{\circ}\right)$ static drift which constitutes the far best performance in the printed angle sensor literature (Fig. 3(b) [5], [6], [10], [11]). 100\% of the combined signals in the experiment population showed a significant drop w/to the mean absolute static drifts of the compression and tension sensors, with an average of $65 \%$ drop.

\section{Noise and Disturbance Cancellation}

Similar to the static drift, circuitry based noise and disturbances are also observed to cancel each other due their opposite signs after being subjected to the normalization function. Fig. 4 (a.i, ii) shows an axial pattern sensor, which has a lower signal to noise ratio as it experiences a lower resistance change compared with the transversal pattern [11]. Although the individual sensor responses are insufficient in distinguishing the $10^{\circ}$ steps, the combined signal is perfectly unique Fig. 4(a.i) without any extra measures for filtering. In Fig. 4(a.ii) a disturbance effect (slight resistance shift between the $32^{\text {nd }}$ and $34^{\text {th }}$ cycles) is seen to cause the same variation in the individual responses, resulting with an unaffected combined signal.

\section{Cyclic Drift Compensation}

Cyclic drift compensation is analyzed using the data represented in Fig. 5. First two graphs (a.i,ii) present the first 30 cycles of a fabricated sensor pair, providing inference on the deformation and resistance change mechanisms of the printed sensors. Considering the crack based resistance change findings in the printed linear sensors [7], [17], imaged cracks for the tension and compression side folded sensors [11], and 


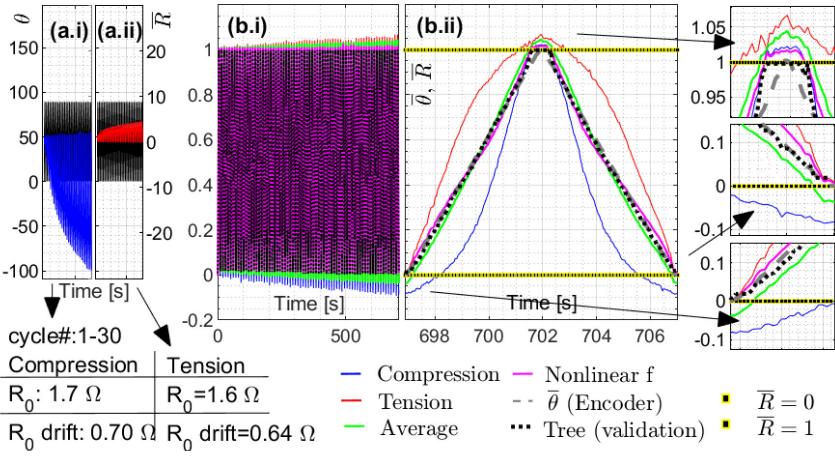

Fig. 5. Cyclic drift compensation performance with linear (average function), nonlinear and regression tree models. (b.ii) shows the last cycle of the data set represented in (b.i). Represented regression tree response is generated with $100 \%$ validation data. Training data is provided as supplementary material.

the captured reversible and irreversible crack formation in the sensor layer (Supplementary Video), deformation history of the sensors indicates the following: Compression side folded sensors tend to have a positive resistance drift at $0^{\circ}$ which can be interpreted as an exponential transition from reversible elastic deformation to irreversible cracks (resulting with less recovery, more \& wider cracks and higher resistance at every turn to the $0^{\circ}$ position). Tension side sensor on the other hand, goes through the same transition at $90^{\circ}$, resulting with a positive resistance drift at every fold. Notice that these drift magnitudes are very close, however the difference of the drifts in the normalized values are very high. This is due to the much higher resistance change (gain) of the tension side folding generates, matching with the longer cracks observed compared with to compression side [11].

Regarding the nearly unchanged resistances of the sensors at the opposite positions, extracting an accurate position information with a single sensor is possible only for one end neighborhood of the folding range. However, thanks to the conjugate operation, a compression-tension pair data contains the accurate information within, for the full range.

Although using an average function is not the best way of extracting this information as it only halves the drift, $3.6^{\circ} \mathrm{drift}$ is achieved at 2400 cycles $\left(0.0015^{\circ} /\right.$ cycle $)$ which is the lowest value in the literature (green curve in Fig. 5(b)). A nonlinear function (4) is tested, in order to weight the more accurate sensor signal at corresponding regions. Fig. 5(b) shows the combined signal eliminated the drifts extensively (maximum $1.8^{\circ}$ drift at 2400 cycles), however resulted with a cubic profile. (It should be noted that for high drift regions this function would give inaccurate values.)

$$
\bar{R}_{\text {combined }}=\operatorname{real}\left(\frac{\bar{R}_{C}^{1.5}-\left(1-\bar{R}_{T}\right)^{1.5}+1}{2}\right)
$$

Finally a regression tree model is trained and validated using the data represented in Fig. 5 for the latter (Model and used data sets are provided in the supplementary material). The most efficient extraction both for drift (maximum $0.9^{\circ} \mathrm{drift}$ at 2400 cycles) and accuracy is achieved with this model, providing a very close response to the linear motor encoder response $\left(0.6^{\circ} \mathrm{RMS}\right)$. This constitutes a record high linearity for any printed flexible angle sensors.

Going back to the average function, experiment population showed a 53\% drop in the overall average drift, with a more significant improvement relative to the compression sensor (61\%), compared with the tension sensor (21\%). Note that these drop values also include noise and disturbance based drift cancellations, which sometimes become more dominant than the deformation based regular drift. Unlike the average model, other two presented approaches do not provide any noise or disturbance cancellation, as no means of subtraction is employed.

\section{E. Hysteresis Compensation}

Although the tension sensors showed a lower hysteresis compared with the compression side (26\% for dynamic hysteresis and 53\% for static hysteresis) no significant regularity is found in the hysteresis shapes of the samples. Consequently number of sample pairs that gave a lower hysteresis RMSE w/to both tension and compression responses remained at 50\% of the whole population. Fig. 4(b) shows an example where the individual hystereses occur in a compensating manner. The average dynamic hysteresis error drop in the combined signals through the population is $36 \%$ w/to compression, $9 \%$ w/to tension and $37 \%$ w/to the mean. Same statistics for the static hysteresis are, $48 \%,-30 \%$ (i.e. an increase) and $32 \%$ respectively. The minimum dynamic hysteresis RMSE achieved with an individual sensor is $0.97^{\circ}$ both for compression and tension sides. With the combined signal a $0.50^{\circ}$ dynamic hysteresis RMSE is achieved (AXL-d1mm-r1mmfixed-h0mm), which is the lowest value in the literature [11]. Similarly the minimum value for the static hysteresis obtained with the combined signals $\left(1.41^{\circ}\right)$ is lower than the best performances of the individuals (compression: $2^{\circ}$, tension: $\left(164^{\circ}\right)$. Note that the hysteresis data collected from the experiment group also contains the noise and nonlinearity effects thus does not provide an ideal data set to analyze the sole effect of the hysteresis.

\section{F. Dynamic Dependency Compensation}

Dynamic dependency of the sensors is measured with the RMSE between the stepwise trajectory steady state responses (average of last $1 \mathrm{sec}$ of each step) to the sharp and smooth profiles (Fig. 4(c.iii)). Fig. 4(c) shows an anisotropic acceleration dependency in the individual sensor responses, in terms of folding directions. This behaviour is the same with reported in [11]. A lower resistance response to the smooth trajectory occurs only at the folding direction for the tension sensor, and only at the unfolding direction for the compression sensor. As the normalized compression sensor signal switches sign, dynamic dependency is partly eliminated (halved) in the combined average signal. $73 \%$ of the experiment population showed lower dynamic dependency (lower RMSE) in the combined signals compared both to the compression and tension sensors. Average drop and the lowest RMSE achieved 
with the combined signals are $34 \%$ and $0.8^{\circ}$ respectively. This result is significant as the dynamic dependency was reported as one of the major causes of tracking control errors [11]. Same coupled composition of the population data stated in the hysteresis compensation section is valid also for the dynamic dependency analysis.

\section{CONCLUSIONS}

Inkjet printed compression-tension sensor pairs are proposed and proved to improve the angle sensing performances at all analyzed metrics. Within the 22 sensor pairs with varying design parameters, combined signals with a simple average function gave better performances than the both individual signals (compression and tension) at over $80 \%$ of the experiment population in terms of nonlinearity, static and cyclic drifts, signal to noise ratio and disturbance rejection. $73 \%$ of the population showed lower dynamic dependency and $50 \%$ showed lower static and dynamic hysteresis. Population average of the combined signal performances on the other hand, are recorded to be higher than the population averages of the both individuals, at all of the metrics. Proposed method advances the existing inkjet printed flexible sensor technology by achieving the best performances of linearity, sensor life-time, static and cyclic drift, hysteresis and dynamic dependency ever recorded in the literature.

Implementation of the proposed approach can be further simplified by using a double side printable substrate \& ink combination. Regarding the fixed-sliding(guided) sensor responses that do not yield the symmetry existing in the fixedfixed(guided) sensors, further analyses will be valuable to reveal the leading mechanisms and design parameters that are shaping the response.

Demonstrated nonlinear and regression tree models for the cyclic drifts showed that a multi-sensor approach for foldable robot hinges can be exploited for even more advanced sensing performances with more complex estimation methods. These advanced models should be studied for wider data sets and performance measures. We believe the compensation approach can enable advanced proprioception and feedback control implementations by resolving the major limiting drawbacks of the printed sensors and eliminate the hard component sensor dependency for the flexible hinges.

\section{REFERENCES}

[1] "The soft touch of robots," Nature Reviews Materials, vol. 3, p. 71, 2018.

[2] B. Ando and S. Baglio, "All-inkjet printed strain sensors," IEEE Sensors Journal, vol. 13, no. 12, pp. 4874-4879, 2013.

[3] Z. Zhakypov and J. Paik, "Design methodology for constructing multimaterial origami robots and machines," IEEE Transactions on Robotics, vol. 34, no. 1, pp. 151-165, 2018.

[4] J. K. Paik, R. K. Kramer, and R. J. Wood, "Stretchable circuits and sensors for robotic origami," in 2011 IEEE/RSJ international conference on intelligent robots and systems. IEEE, 2011, pp. 414-420.

[5] B. Kwak and J. Bae, "Compliant mechanosensory composite (cmc): a compliant mechanism with an embedded sensing ability based on electric contact resistance," Smart Materials and Structures, vol. 27, no. 12 , p. $125003,2018$.
[6] X. Sun, S. M. Felton, R. J. Wood, and S. Kim, "Printing angle sensors for foldable robots," 2015 IEEE/RSJ International Conference on Intelligent Robots and Systems (IROS), 2015.

[7] S. Zhang, L. Cai, W. Li, J. Miao, T. Wang, J. Yeom, N. Sepúlveda, and C. Wang, "Fully printed silver-nanoparticle-based strain gauges with record high sensitivity," Advanced Electronic Materials, vol. 3, no. 7, p. 1700067, 2017.

[8] "A-puffer, a foldable robot that can access tight spaces," https://www.jpl.nasa.gov/robotics-at-jpl/a-puffer, accessed: 2021-07-06.

[9] M. E. Nisser, S. M. Felton, M. T. Tolley, M. Rubenstein, and R. J. Wood, "Feedback-controlled self-folding of autonomous robot collectives," in 2016 IEEE/RSJ International Conference on Intelligent Robots and Systems (IROS). IEEE, 2016, pp. 1254-1261.

[10] A. Firouzeh, A. F. Amon-Junior, and J. Paik, "Soft piezoresistive sensor model and characterization with varying design parameters," Sensors and Actuators A: Physical, vol. 233, pp. 158-168, 2015.

[11] D. Türkmen and M. Acer Kalafat, "Feedback control of a foldable delta mechanism with integrated inkjet-printed angle sensors," 2021, manuscript submitted for publication. Preprint $10.36227 /$ techrxiv. 16688929 .

[12] M. Luo, E. H. Skorina, W. Tao, F. Chen, S. Ozel, Y. Sun, and C. D. Onal, "Toward modular soft robotics: Proprioceptive curvature sensing and sliding-mode control of soft bidirectional bending modules," Soft robotics, vol. 4, no. 2, pp. 117-125, 2017.

[13] M. Mete and J. Paik, "Closed-loop position control of a self-sensing 3-dof origami module with pneumatic actuators," IEEE Robotics and Automation Letters, vol. 6, no. 4, pp. 8213-8220, 2021.

[14] L. Tang, S. Cheng, L. Zhang, H. Mi, L. Mou, S. Yang, Z. Huang, X. Shi, and $X$. Jiang, "Printable metal-polymer conductors for highly stretchable bio-devices," IScience, vol. 4, pp. 302-311, 2018.

[15] V. Correia, C. Caparros, C. Casellas, L. Francesch, J. Rocha, and S. Lanceros-Mendez, "Development of inkjet printed strain sensors," Smart Materials and Structures, vol. 22, no. 10, p. 105028, 2013.

[16] Č. Žlebič, L. Živanov, A. Menićanin, N. Blaž, and M. Damnjanović, "Inkjet printed resistive strain gages on flexible substrates," Facta universitatis-series: Electronics and Energetics, vol. 29, no. 1, pp. 89$100,2016$.

[17] C. T. Gentile, M. Wallace, T. D. Avalon, S. Goodman, R. Fuller, and T. Hall, "Angular displacement sensors," Feb 1992.

[18] J. Lewis, "Material challenge for flexible organic devices," Materials today, vol. 9, no. 4, pp. 38-45, 2006.

[19] C. Beisteiner and B. G. Zagar, "A survey of inkjet-printed low-cost sensors," tm-Technisches Messen, vol. 85, no. 7-8, pp. 504-514, 2018.

[20] M. A. M. Asri, N. A. Ramli, and A. N. Nordin, "Electrical performance and reliability assessment of silver inkjet printed circuits on flexible substrates," Journal of Materials Science: Materials in Electronics, pp. $1-14,2021$.

[21] D. Türkmen and M. Acer Kalafat, "Lamination curing method for inkjet printed flexible angle sensors," 2021, manuscript submitted for publication. Preprint 10.36227/techrxiv.16776145.

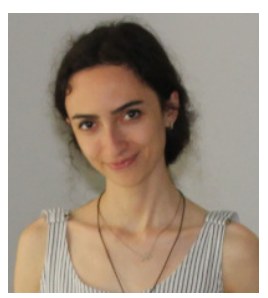

Dila Türkmen is a Ph.D. student and Research Assistant in Istanbul Technical University (ITU), Mechanical Engineering Department, where she completed her B.S. and and M.S. degrees in 2013 and 2016 respectively.

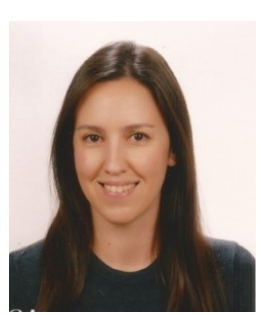

Dr. Merve Acer Kalafat received her B.S. in Mechanical Engineering from ITU (2005), and her M.S $\&$ Ph.D. in Mechatronics Engineering from Sabanci University (2007 \& 2012). She worked as a postdoc in EPFL at Reconfigurable Robotics Laboratory (2013-2014). She is an instructor in the Mechanical Engineering Department at ITU since 2012, where she established Flexible Systems Laboratory in 2018 . 